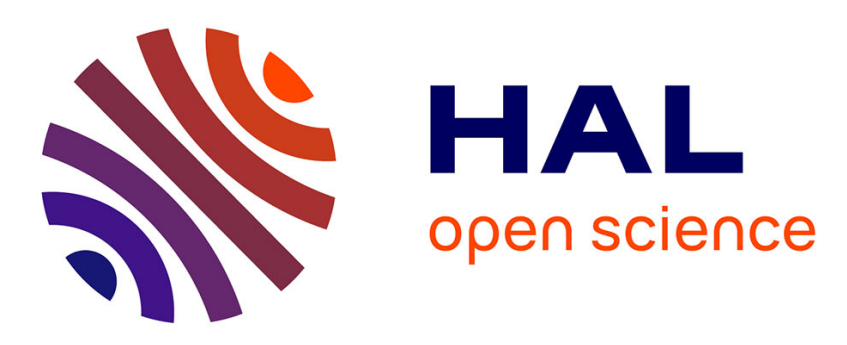

\title{
Effective model for a short Josephson junction with a phase discontinuity
}

E. Goldobin, S. Mironov, A. Buzdin, R. G. Mints, D. Koelle, R. Kleiner

\section{To cite this version:}

E. Goldobin, S. Mironov, A. Buzdin, R. G. Mints, D. Koelle, et al.. Effective model for a short Josephson junction with a phase discontinuity. Physical Review B: Condensed Matter and Materials Physics (1998-2015), 2016, 93 (13), pp.134514 (1-10). 10.1103/PhysRevB.93.134514 . hal-01342343

\section{HAL Id: hal-01342343 \\ https://hal.science/hal-01342343}

Submitted on 5 Jul 2016

HAL is a multi-disciplinary open access archive for the deposit and dissemination of scientific research documents, whether they are published or not. The documents may come from teaching and research institutions in France or abroad, or from public or private research centers.
L'archive ouverte pluridisciplinaire HAL, est destinée au dépôt et à la diffusion de documents scientifiques de niveau recherche, publiés ou non, émanant des établissements d'enseignement et de recherche français ou étrangers, des laboratoires publics ou privés. 


\title{
Effective model for a short Josephson junction with a phase discontinuity
}

\author{
E. Goldobin, ${ }^{1, *}$ S. Mironov, ${ }^{2, \dagger}$ A. Buzdin, ${ }^{2, \dagger}$ R. G. Mints, ${ }^{3, \S}$ D. Koelle, ${ }^{1, \|}$ and R. Kleiner ${ }^{1, \mathbb{y}}$ \\ ${ }^{1}$ Physikalisches Institut and Center for Quantum Science in LISA ${ }^{+}$, Universität Tübingen, \\ Auf der Morgenstelle 14, D-72076 Tübingen, Germany \\ ${ }^{2}$ LOMA, UMR-CNRS 5798, Université Bordeaux, 351, cours de la Liberation, F-33405 Talence Cedex, France \\ ${ }^{3}$ The Raymond and Beverly Sackler School of Physics and Astronomy, Tel Aviv University, Tel Aviv 69978, Israel
}

(Received 17 August 2015; revised manuscript received 18 March 2016; published 25 April 2016)

\begin{abstract}
We consider a short Josephson junction with a phase discontinuity $\kappa$ created, e.g., by a pair of tiny current injectors, at some point $x_{0}$ along the width of the junction. We derive the effective current-phase relation (CPR) for the system as a whole, i.e., reduce it to an effective pointlike junction. From the effective CPR we obtain the ground state of the system and predict the dependence of its critical current on $\kappa$. We show that in a large range of $\kappa$ values the effective junction behaves as a $\varphi_{0}$ Josephson junction, i.e., has a unique ground state phase $\varphi_{0}$ within each $2 \pi$ interval. For $\kappa \approx \pi$ and $x_{0}$ near the middle of the junction one obtains a $\varphi_{0} \pm \varphi$ junction, i.e., a Josephson junction with degenerate ground state phase $\varphi_{0} \pm \varphi$ within each $2 \pi$ interval. Further, in view of possible escape experiments especially in the quantum domain, we investigate the scaling of the energy barrier and eigenfrequency close to the critical currents and predict the behavior of the escape histogram width $\sigma(\kappa)$ in the regime of the macroscopic quantum tunneling.
\end{abstract}

DOI: 10.1103/PhysRevB.93.134514

\section{INTRODUCTION}

Conventional Josephson junctions superconductor-insulator-superconductor superconductor-normal metal-superconductor (SNS) JJs, have a current-phase relation (CPR) (the first Josephson relation), which assumes zero phase drop $\phi$ across the JJ when no supercurrent $I_{s}$ flows through the JJ. The most typical and widely applicable CPR of this type is $I_{s}=I_{c} \sin (\phi)$, where $I_{c}>0$ is the critical current (the maximum supercurrent that can flow through the JJ). In other words, the Josephson energy $U_{J}(\phi)$ has a minimum at $\phi=0$. In the context of novel and unconventional JJs (see below), such conventional JJs with $\phi=0$ in the ground state (i.e., when no current is applied) are called "0 JJs."

Presently a lot of attention is attracted to Josephson junctions (JJs) with an unconventional current-phase relation (CPR) [1,2], especially those having the ground state phase $\phi \neq 2 \pi n$ ( $n$ is any integer). Historically, it started from $\pi$ JJs $[3,4]$, where the CPR is also sine-like, but with $I_{c}<0$. This turns the Josephson energy minimum at $\phi=0$ into maximum and the maximum at $\phi=\pi$ into minimum. Starting from 2001, such $\pi$ JJs were demonstrated by several groups on the base of superconductor-insulator-ferromagnet-superconductor (SIFS) [5-7] or SFS [8-11] heterostructures as well as using quantum dots JJs [12]. Nowadays researchers proposed and/or even demonstrated $\varphi_{0}$ JJs [13-18], $\varphi$ JJs [19-25] and their combinations [17]. Such JJs show nontrivial physics [26] and have potential for applications in the classical [27-29] and

\footnotetext{
*gold@uni-tuebingen.de

†Present address: Moscow Institute of Physics and Technology, 147700, Dolgoprudny, Russia; SerMironov@ rambler.ru

${ }^{\ddagger}$ Alexandre.Bouzdine@u-bordeaux.fr

$\S$ Mints@post.tau.ac.il

"Koelle@uni-tuebingen.de

"Kleiner@uni-tuebingen.de
}

the quantum domains [30]. Here, $\varphi_{0} \mathrm{JJ}$ s are defined as $\mathrm{JJ}_{\mathrm{S}}$ having a unique ground state phase (single Josephson energy minimum situated at) $\varphi_{0} \neq 0$ within each $2 \pi$ phase interval, while $\varphi \mathrm{JJS}$ (sometimes denoted also $\pm \varphi \mathrm{JJ}$ ) have a doubly degenerate ground state phase (double-well Josephson energy with minima at) $\pm \varphi$ within each $2 \pi$ interval.

Currently, the classical properties of $\varphi \mathrm{JJ}$ s made of a short $0-\pi \mathrm{JJ}$ are understood rather well $[22,23,29]$. For example, $\varphi$ JJs have two critical currents $I_{c-}$ and $I_{c+}$ corresponding to the escape of the phase from $-\varphi$ and $+\varphi$ wells of $U_{J}(\phi)$. In our group we are starting investigation of quantum properties of such JJs. The first step in this direction could be an observation of the macroscopic quantum tunneling (MQT) of the phase [31-33] out of both $-\varphi$ and $+\varphi$ wells of the Josephson energy profile. For this purpose, one, usually, measures the phase escape statistics by sweeping the bias current at a constant rate and measuring the exact value of the switching current many times. Assuming that in $\varphi \mathrm{JJ}$ at low temperatures (low damping) the initial state $(-\varphi$ or $+\varphi$ ) is random [26], the switching current histogram should have two peaks, each of them just below the corresponding critical current $I_{c \pm}$. The widths $\sigma(T)$ of each histogram peak usually (when the damping is small) decreases with decreasing temperature $T$. However, $\sigma(T)$ is expected to saturate at some value $\sigma_{\min }$ for temperature below some $T^{*}$. Such behavior is usually interpreted as a transition from the regime of the thermal activation of the phase over the barrier to the regime of the MQT of the phase through the barrier. However, it is necessary to show that the observed $\sigma_{\min }$ is not related to the noise in the experimental setup and other trivial reasons. Usually, in such experiments one introduces some extra tuning parameter, e.g., a magnetic field, which allows us to demonstrate that the setup is able to measure the histograms that are more narrow than $\sigma_{\min }$. Simultaneously, for the MQT experiment with $\varphi \mathrm{JJ}$, it would be advantageous to have a tuning parameter, which provides a continuous transition between $\varphi$ (or $\varphi_{0}$ ) JJ and a conventional $0 \mathrm{JJ}$, whose physics is well studied. 


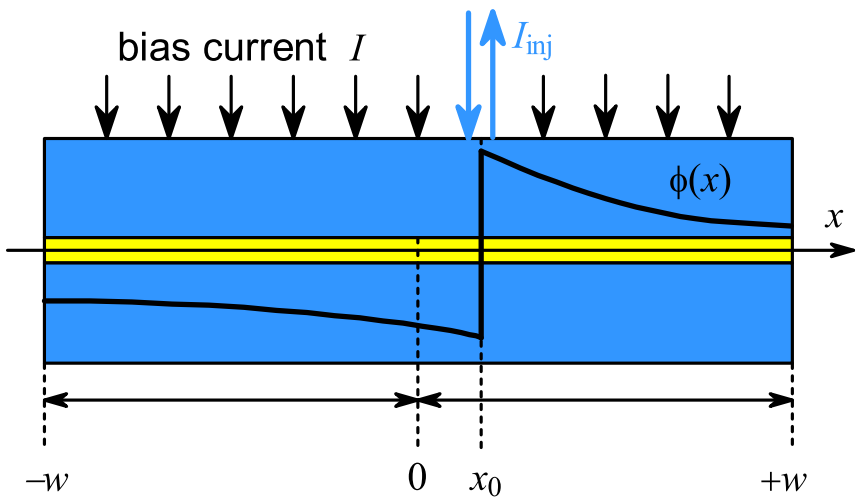

FIG. 1. Geometry of the system: long Josephson junction of width $2 w$ with an infinitesimal pair of current injectors situated at $x=x_{0}$. The bias current density $j$ is applied uniformly along the junction.

For this purpose, we propose to use a setup shown in Fig. 1, i.e., a conventional $0 \mathrm{JJ}$, with a moderate width $2 w$ in $x$ direction. In addition, the $\mathrm{JJ}$ is equipped with a pair of tiny current injectors, see Fig. 1. By sending a current $I_{\text {inj }}$ from one injector to the other, we can create a $\kappa$ discontinuity of the Josephson phase at point $x_{0}$, where injectors are attached [34-36]. A similar setup was used in several experiments $[37,38]$. If $\kappa=\pi$, the system is similar to a superconductor-insulator-ferromagnet-superconductor (SIFS) $0-\pi \mathrm{JJ}$, which becomes a $\varphi \mathrm{JJ}$, if parameters are chosen correctly $[39,40]$. However, since $\kappa \propto I_{\text {inj }}$ is adjustable, one can in situ tune the junction from a $0 \mathrm{JJ}$ to a $\varphi \mathrm{JJ}$ and also study all the states in between. Tuning $\kappa$ one can also affect the widths $\sigma_{\min }$ of the histograms.

The aim of this paper is to develop a theoretical model for a short $\mathrm{JJ}$ with $\kappa$ discontinuity of the phase and to predict or interpret the results of MQT experiment such as the one outlined above. Namely, we derive an effective (averaged) CPR for a short JJ with a phase discontinuity $\kappa$ and obtain experimentally relevant quantities, such as the critical current or the escape histogram width as a function of $\kappa$.

The paper is organized as follows. In Sec. II we introduce the model and present the averaged CPR and the averaged Josephson energy derived in details in Appendix A. In Sec. III we obtain several experimentally relevant dependences such as the ground state phase, critical current and escape-related characteristics as functions of $\kappa$. Sec. IV concludes the work.

\section{MODEL}

We consider a JJ shown in Fig. 1. Its width $2 w$ in $x$ direction is smaller than the Josephson length $\lambda_{J}$. For our analysis we use normalized units. Thus, $2 w<1$ in units of $\lambda_{J}$. In $y$ direction (out of figure plane) the $\mathrm{JJ}$ is even more narrow. The Josephson phase $\phi(x)$ changes along the $x$ coordinate $(-w<x<+w)$. At $x=x_{0}\left(-w<x_{0}<+w\right)$ there is a $\kappa$ discontinuity of the Josephson phase, created, e.g., by a pair of tiny (in theory infinitesimal) current injectors [41]. The junction is biased by a uniform current density $j$. Below we use normalized bias current density $\gamma=j / j_{c}$ given in the units of the critical current density $j_{c}$. Our aim is to derive an effective (averaged over the JJ width) current-phase relation for this system, i.e., $\gamma(\psi)$, where

$$
\psi \equiv\langle\phi(x)\rangle=\frac{1}{2 w} \int_{-w}^{+w} \phi(x) d x,
$$

is the average phase across the JJ. It is $\psi$ that is actually measured, if one considers the system described above as a black box with two electrodes.

In Appendix A we derive the averaged CPR of the system under question by using the perturbation theory up to the second order in $w$, treating $w$ (the half-width of the $\mathbf{J J}$ ) as a small parameter. It is convenient to write the resulting averaged CPR as function of the phase $\theta$, which is related to the average phase $\psi$ across the $\mathrm{JJ}$ as

$$
\theta=\psi+\frac{\kappa}{2} X_{0}
$$

where $X_{0}=x_{0} / w$. The averaged CPR can be written as a Taylor-expansion

$$
\gamma(\theta)=\gamma_{0}(\theta)+w^{2} \gamma_{2}(\theta)+O\left(w^{4}\right)
$$

where

$$
\gamma_{0}(\theta)=\cos \left(\frac{\kappa}{2}\right) \sin (\theta)-X_{0} \sin \left(\frac{\kappa}{2}\right) \cos (\theta),
$$

is the 0th order result of the perturbation theory, the first order gives no correction, and

$$
\gamma_{2}(\theta)=\frac{Q}{w^{2}} \sin ^{2}\left(\frac{\kappa}{2}\right) \sin (2 \theta)
$$

is the second order correction. The quantity $Q=\left(w^{2} / 6\right)$ $\left(1-X_{0}^{2}\right)^{2}$ is introduced to make some formulas below more compact.

The effective Josephson energy of the system is an integral of the effective CPR (3) and is given by

$$
U_{J}(\theta)=U_{J 0}(\theta)+w^{2} U_{J 2}(\theta)+O\left(w^{4}\right),
$$

where

$$
\begin{gathered}
U_{J 0}(\theta)=-\cos \left(\frac{\kappa}{2}\right) \cos (\theta)-X_{0} \sin \left(\frac{\kappa}{2}\right) \sin (\theta) ; \\
U_{J 2}(\theta)=-\frac{Q}{2 w^{2}} \sin ^{2}\left(\frac{\kappa}{2}\right) \cos (2 \theta) .
\end{gathered}
$$

In Appendix B we show that in the particular case $\kappa=\pi$ our results coincide with those obtained earlier for an asymmetric $0-\pi$ JJ [39].

\section{RESULTS}

\section{A. Ground state phase and the critical current}

In the 0th approximation the averaged CPR (4) can be rewritten as

$$
\gamma_{0}=\gamma_{c 0}(\kappa) \sin \left(\theta-\theta_{0}\right)=\gamma_{c 0}(\kappa) \sin \left(\psi-\varphi_{0}\right),
$$

where

$$
\gamma_{c 0}(\kappa)=\sqrt{X_{0}^{2} \sin ^{2}\left(\frac{\kappa}{2}\right)+\cos ^{2}\left(\frac{\kappa}{2}\right)}
$$

is the maximum supercurrent. The critical current measured in experiment is $\pm \gamma_{c 0}(\kappa) . \gamma_{c 0}(\kappa)$ has maxima equal to 1 at 

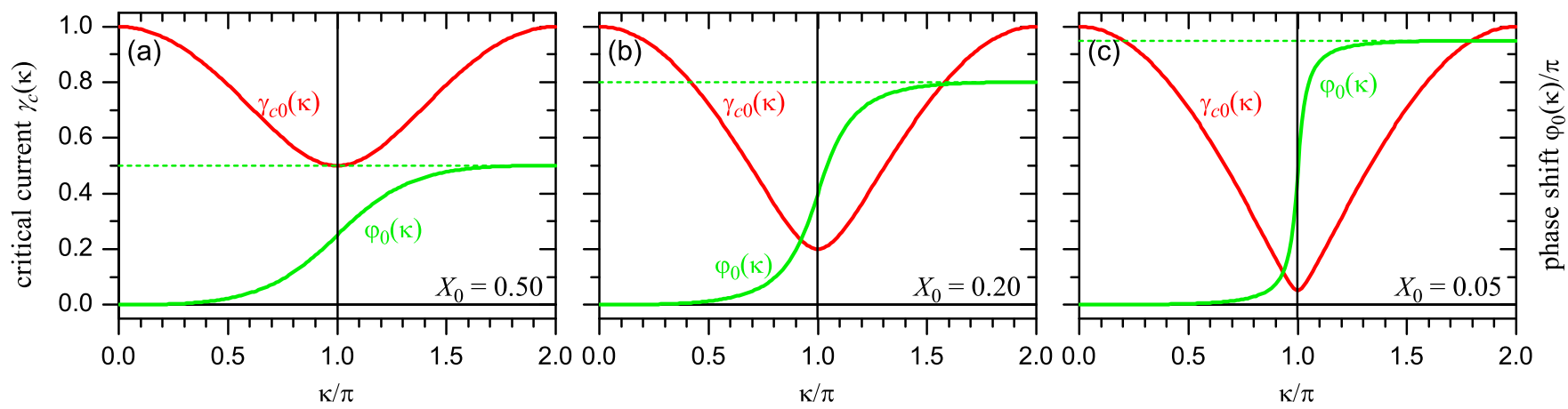

FIG. 2. The $\gamma_{c 0}(\kappa)$ and $\varphi_{0}(\kappa)$ curves calculated using Eqs. (10) and (11) for (a) $X_{0}=0.5$, (b) $X_{0}=0.2$, and (c) $X_{0}=0.05$.

$\kappa=2 \pi n$ ( $n$ is any integer) and minima equal to $X_{0}$ at $\kappa \bmod$ $2 \pi=\pi$, see Fig. 2 .

In Eq. (9) the ground state phase $\varphi_{0}=\theta_{0}-X_{0} \kappa / 2$, where

$$
\theta_{0}=\arg \left[\cos \left(\frac{\kappa}{2}\right)+i \cdot X_{0} \sin \left(\frac{\kappa}{2}\right)\right],
$$

and the function $\arg (z)$ returns the $\operatorname{argument}$ (phase angle) of a complex number $z$. Obviously, the CPR given by Eq. (9) corresponds to a $\varphi_{0} \mathrm{JJ}$ [13-18]. Some examples of $\gamma_{c 0}(\kappa)$ and $\varphi_{0}(\kappa)$ dependences are shown in Fig. 2. For large asymmetry $X_{0}$ the modulation of $\gamma_{c 0}(\kappa)$ is not as deep as for $X_{0} \rightarrow 0$. The phase shift $\varphi_{0}(\kappa)$ changes from 0 to $\pi\left(\operatorname{sgn}\left(X_{0}\right)-X_{0}\right)$ as $\kappa$ changes from 0 to $2 \pi$. It is positive for $X_{0}>0$ and negative for $X_{0}<0$.

When $X_{0} \rightarrow 0$, the critical current given by the 0 th order formula (10) vanishes close to $\kappa=\pi$ and one has to take into account the next (second) order corrections given by Eq. (5). This happens for $X_{0} \lesssim w^{2} / 3$, see the discussion after Eq. (B7).

Next, we consider the second order approximation. The ground state phase $\theta_{\mathrm{gs}}(\kappa)$ is a solution of $\gamma\left(\theta_{\mathrm{gs}}\right)=0$ for this value of $\kappa$, where $\gamma(\theta)$ is given by Eqs. (3), (4), and (5). This equation can be solved only numerically, see Fig. 3. It can be seen that multiple solutions $\theta_{\mathrm{gs}}(\kappa)$ appear in the vicinity of $\kappa=\pi$, see Figs. 3(b) and 3(c). To find the approximate analytical expression describing them we take $\kappa=\pi+\delta \kappa$ $(|\delta \kappa| \ll 1)$. Then we expand the equation $\gamma\left(\theta_{\mathrm{gs}}\right)=0$, up to the first order in $\delta \kappa$ and solve it for $\delta \kappa$. Finally we obtain an approximate value of $\kappa$ for any given ground state phase $\theta_{\mathrm{gs}}$,

$$
\kappa\left(\theta_{\mathrm{gs}}\right) \approx \pi+2\left[2 Q \cos \left(\theta_{\mathrm{gs}}\right)-X_{0} \cot \left(\theta_{\mathrm{gs}}\right)\right],
$$

i.e., the inverse of the ground state phase $\theta_{\mathrm{gs}}(\kappa)$. This approximation is also shown in Fig. 3. We see that the approximation given by Eq. (12) is very good in the whole range of $0<\kappa<2 \pi$. Note, that the appearance of three solutions (two stable and one unstable) out of one near $\kappa=\pi$ is a result of the competition of the cos term with the cot term in Eq. (12). From Eq. (12) one can figure out that the multiple solutions appear for $X_{0}<2 Q$, i.e., $\left|X_{0}\right|<w^{2} / 3$, which is in agreement with the discussion after Eq. (B7). We note that numerical solution of $\gamma\left(\theta_{\mathrm{gs}}\right)=0$ or Eq. (12) give just static solutions. To select which of them corresponds to the energy minimum (ground state), we should analyze $U^{\prime \prime}(\theta)$ for each of them. The results are presented in Fig. 3. One can see that only the parts of $\theta_{\mathrm{gs}}(\kappa)$ colored in green (having the positive slope and $0<\theta_{\mathrm{gs}}<\pi$ ) are stable (energy minima) and can indeed be called the ground state.

From Eq. (12) one can find the range of $\kappa$ where the double ground state exists, i.e., the points $\theta_{1}$ and $\theta_{2}$ in Fig. 3 where $d \kappa\left(\theta_{\mathrm{gs}}\right) / d \theta_{\mathrm{gs}}=0$. We obtain that

$$
\theta_{1}=\arcsin \sqrt[3]{\frac{X_{0}}{2 Q}} \text { and } \theta_{2}=\pi-\arcsin \sqrt[3]{\frac{X_{0}}{2 Q}},
$$

which lay symmetrically with respect to $\theta=\pi / 2$. It follows from Eqs. (13) that the bifurcation point, where the $\theta(\kappa)$ curve
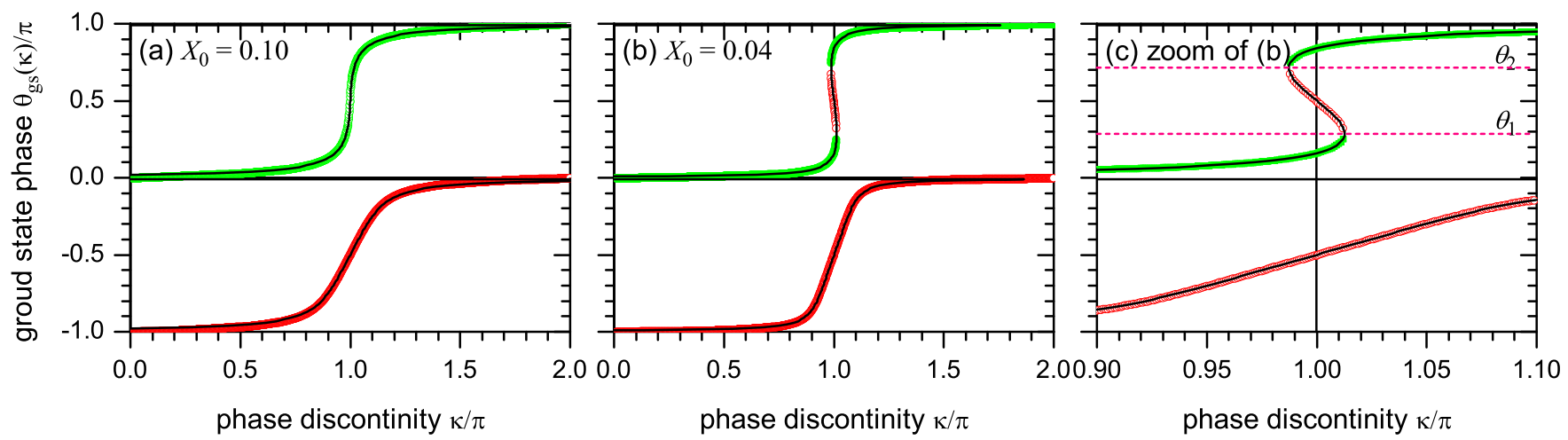

FIG. 3. The ground state phase $\theta_{\mathrm{gs}}(\kappa)$. Comparison of the approximate dependence given by Eq. (12) (black line) with the exact dependence calculated by numerically solving $\gamma(\theta)=0$, see Eq. (3), for each value of $\kappa$ (symbols). (a) $X_{0}=0.10$, (b) $X_{0}=0.04$, (c) $X_{0}=0.04$ and the region close to $\kappa=\pi$ zoomed. In all plots the regions with a positive slope and $0<\theta_{\mathrm{gs}}<\pi$ (green) correspond to a stable solution [energy minimum, where $U^{\prime \prime}(\theta)=\gamma^{\prime}(\theta)>0$ ], while the regions with a negative slope for $0<\theta_{\mathrm{gs}}<\pi$ or any slope for $-\pi<\theta_{\mathrm{gs}}<0$ (red) correspond to an unstable one [energy maximum, where $U^{\prime \prime}(\theta)=\gamma^{\prime}(\theta)<0$ ]. 

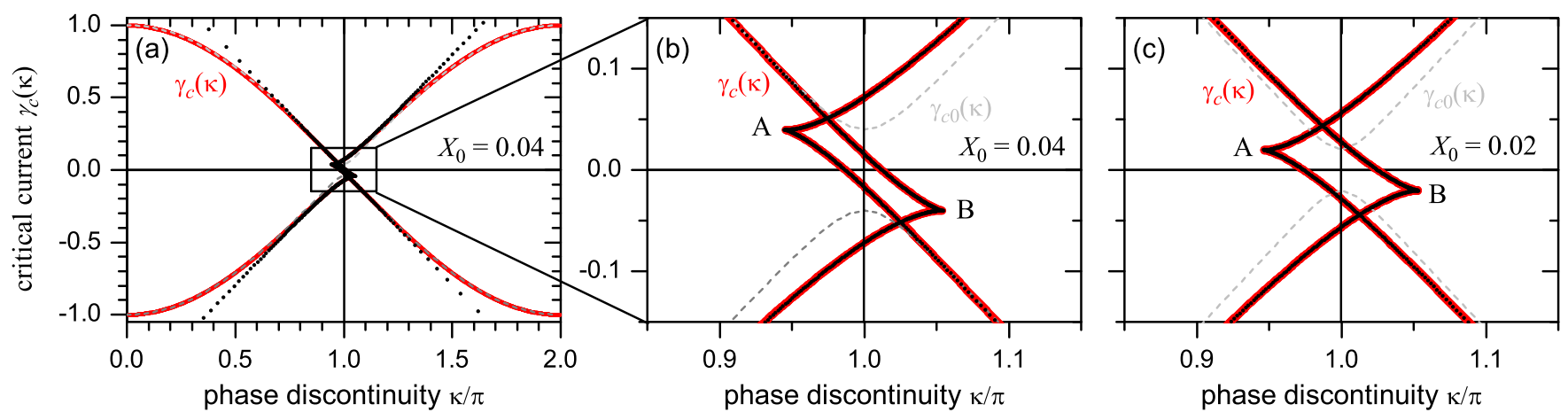

FIG. 4. Examples of the $\gamma_{c}(\kappa)$ dependence for $w=0.5$. (a) $X_{0}=0.04$, global behavior of $\gamma_{c}(\kappa)$; (b) $X_{0}=0.04$, zoom of the region of interest near $\kappa=\pi$; (c) $X_{0}=0.02$, zoom of the region near $\kappa=\pi$. Thick (red) lines/symbols show $\gamma_{c}$ obtained by directly solving Eq. (15) numerically to find all $\theta_{c}$ and then calculating $\gamma_{c}$ from Eq. (3). Thinner black lines/symbols correspond to the approximation given by Eq. (17). Gray dashed lines show $\gamma_{c 0}(\kappa)$, see Eq. (10), for the same parameters.

switches from one stable to three solutions (two stable and one unstable), corresponds to $\theta_{1}=\theta_{2}=\pi / 2$, i.e., at $X_{0}=2 Q \approx$ $w^{2} / 3$, which is again in agreement with the result obtained directly from Eq. (12). The range of $\kappa$ around $\kappa=\pi$ where two stable solutions exist, is found by substituting Eq. (13) into Eq. (12).

Looking at the Fig. 3 one sees that the ground state phase $\theta_{\mathrm{gs}}$ has the values symmetrically placed around $\theta=$ $(\pi / 2) \operatorname{sgn}\left(X_{0}\right)$ only at $\kappa=\pi$. From Eq. (12) they are given by

$$
\theta_{\mathrm{gs} 1}(\pi)=\arcsin \left[\frac{X_{0}}{2 Q}\right] \text { and } \theta_{\mathrm{gs} 2}(\pi)=\pi-\arcsin \left[\frac{X_{0}}{2 Q}\right] \text {. }
$$

For $\kappa \neq \pi$ the symmetry is broken because the corresponding double-well potential Eq. (6) becomes asymmetric (one well is deeper than the other) relative to $\theta=(\pi / 2) \operatorname{sgn}\left(X_{0}\right)$. The real (measurable) ground state phases are given by $\psi_{\mathrm{gs}}=$ $\theta_{\mathrm{gs}}-X_{0} \kappa / 2$ and at $\kappa=\pi$ are symmetric with respect to the phase $(\pi / 2)\left[\operatorname{sgn}\left(X_{0}\right)-X_{0}\right]$. Recalling that for doubly generate state to occur one needs very small $\left|X_{0}\right| \lesssim w^{2}$, the shift from $(\pi / 2) \operatorname{sgn}\left(X_{0}\right)$ is small. Thus, such a JJ can be called a $\varphi_{0} \pm \varphi \mathrm{JJ}$, where, at $\kappa=\pi \varphi_{0}=(\pi / 2)\left[\operatorname{sgn}\left(X_{0}\right)-X_{0}\right]$ and $\varphi=\pi / 2-\arcsin \left(X_{0} / 2 Q\right)$.

To find the critical current(s) in the second order approximation for each $\kappa$ we search for an extremum of $\gamma(\theta, \kappa)$ with respect to $\theta$. It takes place at $\theta=\theta_{c}$ for which

$$
\begin{aligned}
\gamma^{\prime}\left(\theta_{c}, \kappa\right)= & X_{0} \sin \left(\frac{\kappa}{2}\right) \sin \left(\theta_{c}\right)+\cos \left(\frac{\kappa}{2}\right) \cos \left(\theta_{c}\right) \\
& +2 Q \sin ^{2}\left(\frac{\kappa}{2}\right)\left[2 \cos ^{2}\left(\theta_{c}\right)-1\right]=0 .
\end{aligned}
$$

Here and below the prime denotes $\partial / \partial \theta$. This equation can be solved for $\theta_{c}$ only numerically to find several (up to 4) $\theta_{c}$ for each value of $\kappa$. Then we substitute each of these $\theta_{c}$ into Eq. (3) to find $\gamma_{c}(\kappa)=\gamma\left(\theta_{c}, \kappa\right)$. The result is presented in Fig. 4. The global behavior is defined mainly by $\gamma_{0}$, i.e., $\gamma_{c 0}$. However, near $\kappa=\pi$, where $\gamma_{c 0}$ vanishes, $\gamma_{2}$ results in a bistability and in the formation of a $\diamond$-like intersection of the branches. Such $\gamma_{c}$ behavior is typical for a $\varphi \mathrm{JJ}$ made of 0 and $\pi$ parts $[17,39,40]$.
Similar to the case of the ground state phase, one can find an approximate expression for $\gamma_{c}(\kappa)$ near $\kappa=\pi$. By substituting $\kappa=\pi+\delta \kappa(|\delta \kappa| \ll 1)$ into Eq. (15), Taylor-expanding it up to terms $O(\delta \kappa)$, and expressing $\delta \kappa$, we obtain the critical value of $\kappa$ corresponding to $\gamma_{c}$ for given $\theta$ :

$$
\kappa_{c}(\theta)=\pi+\delta \kappa \approx \pi+2 \frac{X_{0} \sin (\theta)+2 Q \cos (2 \theta)}{\cos (\theta)} .
$$

To calculate the critical current, we substitute Eq. (16) into Eq. (3), which was preliminary expanded near $\kappa=\pi$ up to $O(\delta \kappa)$. We obtain

$$
\begin{aligned}
\gamma_{c}^{\mathrm{ap}}(\theta) \approx & -X_{0} \cos (\theta)+Q \sin (2 \theta)- \\
& -\frac{X_{0} \sin ^{2}(\theta)+2 Q \sin (\theta) \cos (2 \theta)}{\cos (\theta)} .
\end{aligned}
$$

By sweeping $\theta$ in the range $-\pi \ldots \pi$, we can now calculate $\kappa_{c}(\theta)$ and $\gamma_{c}^{\mathrm{ap}}(\theta)$ and make a parametric plot of $\gamma_{c}^{\mathrm{ap}}(\theta) v s . \kappa_{c}(\theta)$, see Fig. 4. The agreement with direct numerical calculations near $\kappa=\pi$ is excellent, see Figs. 4(b) and 4(c). The deviations become noticeable as $\kappa \bmod 2 \pi$ approaches 0 or $2 \pi$, see Fig. 4(a).

This approximate analytical expression for $\gamma_{c}$ allows us to calculate some key features in the $\gamma_{c}(\kappa)$ plot. For example, one can find out the value of $\theta_{\diamond}$ (and $\kappa_{\diamond}$ ), for which the branches meet each other, see points A and B in Fig. 4. The analysis of the $\kappa_{c}(\theta)$ dependence (16) shows that this happens when $d \kappa_{c} / d \theta=0$. Differentiating Eq. (16) we obtain the following equation for $\theta_{\diamond}$.

$$
4 Q \sin ^{3}\left(\theta_{\diamond}\right)-6 Q \sin \left(\theta_{\diamond}\right)+X_{0}=0 .
$$

This cubic equation with respect to $\sin \left(\theta_{\diamond}\right)$ has only one suitable root, which (after some lengthy algebra) can be expressed as

$$
\sin \left(\theta_{\diamond}\right)=\frac{-1}{\sqrt{2}}\left[\cos \left(\frac{\chi}{3}\right)-\sqrt{3} \sin \left(\frac{\chi}{3}\right)\right],
$$

where $\chi$ may be explicitly written as

$$
\begin{gathered}
\chi=\arg \left[-X_{0}+i \cdot \sqrt{8 Q^{2}-X_{0}^{2}}\right] . \\
\kappa_{\diamond} \approx \pi \pm\left[\frac{2}{3} w^{2}+\frac{X_{0}^{2}}{w^{2}}\right] .
\end{gathered}
$$




\section{B. Energy barrier}

We consider the thermal escape or the quantum tunneling of the phase $\theta$ out of the potential well, when the bias current $\gamma \rightarrow \gamma_{c}(\kappa)$. Since our model reduces the system to an effective pointlike $J J$, for calculation of the escape rate $\Gamma$ one can use standard thermal or quasiclassical quantum formulas. In these formulas, the key parameters are the barrier height $\Delta U(\gamma)$ and the eigenfrequency $\omega_{0}(\gamma)$. The aim of this section is to obtain the expressions for them.

In general, we proceed as follows. Given the Josephson energy profile $U_{J}(\theta)$, the total potential energy of the biased $\mathrm{JJ}$ can be written as a tilted potential

$$
U(\theta)=U_{J}(\theta)-\gamma \theta .
$$

The static solution(s) correspond(s) to

$$
U_{J}^{\prime}(\theta)-\gamma=0,
$$

In essence this is a CPR. The critical current is reached for $\theta=\theta_{c}$, when $d \gamma / d \theta=0$, i.e.,

$$
U_{J}^{\prime \prime}\left(\theta_{c}\right)=0 .
$$

From here one can, in principle, find (one or more) values of $\theta_{c}$. Imagine that we have found all values of $\theta_{c}$. Then, the value of the critical current $\gamma_{c}$ is found from Eq. (23), i.e.,

$$
\gamma_{c}=U_{J}^{\prime}\left(\theta_{c}\right) \text {. }
$$

Now we assume that the value of $\gamma$ is slightly undercritical, i.e., $\gamma=\gamma_{c}(1-\delta \gamma)$, where $0 \leqslant \delta \gamma \ll 1$ for any sign of $\gamma_{c}$. In this case, the minimum of the potential is situated at $\theta=\theta_{c}+\delta \theta$ $(|\delta \theta| \ll 1)$, where

$$
\delta \theta=\sqrt{\frac{-2 \gamma_{c} \delta \gamma}{U_{J}^{\prime \prime \prime}\left(\theta_{c}\right)}}
$$

to the positive or negative direction. One of them is stable and corresponds to the minimum of $U(\theta)$; another unstable one corresponds to the maximum of $U(\theta)$. The energy barrier

$$
\Delta U=\left|U\left(\theta_{c}+\delta \theta\right)-U\left(\theta_{c}-\delta \theta\right)\right| .
$$

After expanding we obtain

$$
\Delta U=\left|2 U_{J}^{\prime}\left(\gamma_{c}\right) \delta \theta-2 \gamma \delta \theta+\frac{2}{6} U_{J}^{\prime \prime \prime}\left(\theta_{c}\right) \delta \theta^{3}\right| .
$$

Using Eq. (25), the definition of $\gamma=\gamma_{c}(1-\delta \gamma)$ and the expression (26), we finally obtain

$$
\Delta U=\frac{4 \sqrt{2}}{3} \frac{\left|\gamma_{c} \delta \gamma\right|^{3 / 2}}{\sqrt{\left|U_{J}^{\prime \prime \prime}\left(\theta_{c}\right)\right|}}=\frac{4 \sqrt{2}}{3} \sqrt{\left|\frac{\gamma_{c}^{3}}{U_{J}^{\prime \prime \prime}\left(\theta_{c}\right)}\right|} \delta \gamma^{3 / 2} .
$$

Now let us apply this general result to our system.

If $X_{0} \gg w^{2} / 3$ or if $\kappa$ is far away from $\kappa=\pi$, then we can use only the 0th order term in Eq. (3) and in Eq. (6). In this limit the CPR is sinusoidal, see Eq. (9). Although it is shifted by $\theta_{0}\left(\varphi_{0}\right)$, it is irrelevant for calculation of the escape barrier and eigenfrequency. Thus, the system behaves as a conventional JJ with sinusoidal CPR, critical current $\gamma_{c 0}(\kappa)$ and Josephson energy $U_{J}=\gamma_{c 0}(\kappa)\left[1-\cos \left(\theta-\theta_{0}\right)\right]$. Thus, $\theta_{c}=$ $\pi / 2, U^{\prime \prime \prime}\left(\theta_{c}\right)=-\gamma_{c 0}$ and we obtain the usual approximation for the energy barrier and the eigenfrequency in the limit $\gamma \rightarrow$ $\pm \gamma_{c 0}$ :

$$
\Delta U(\delta \gamma, \kappa)=\frac{4 \sqrt{2}}{3} \gamma_{c 0}(\kappa) \delta \gamma^{3 / 2} .
$$

In the case when the second order correction is important, i.e., $X_{0} \lesssim w^{2} / 3$ and $\kappa \approx \pi$, we use the same approach, but again, like in the section about critical current and ground state phase, we approximate for $\kappa=\pi+\delta \kappa(|\delta \kappa| \ll 1)$. In this case the energy is given by

$$
\begin{gathered}
U(\theta, \delta \kappa)=\frac{\delta \kappa}{2} \cos (\theta)-X_{0} \sin (\theta)-\frac{Q}{2} \cos (2 \theta) . \\
U^{\prime \prime \prime}(\theta, \kappa)=\frac{\kappa-\pi}{2} \sin (\theta)+X_{0} \cos (\theta)-4 Q \sin (2 \theta) .
\end{gathered}
$$

Then we calculate $U^{\prime \prime \prime}\left(\theta_{c}\right)$ and substitute it into Eq. (29). The dependence of $\Delta U$ on $\delta \gamma$ is obvious from Eq. (29), so our aim is to see how the prefactor in Eq. (29) depends on $\kappa$ (or $\delta \kappa$ ). Since $\theta_{c}$ for each $\kappa$ can be found only numerically, we, as in the previous sections, sweep $\theta$ from $-\pi$ to $+\pi$ and find the corresponding $\kappa_{c}(\theta)$ from Eq. (16) and then calculate $U^{\prime \prime \prime}\left(\theta, \kappa_{c}(\theta)\right)$ from Eq. (32). Then we make a parametric plot of the energy barrier prefactor

$$
P_{\Delta U}(\theta) \equiv \frac{\Delta U\left(\theta, \kappa_{c}(\theta)\right)}{\delta \gamma^{3 / 2}}=\frac{4 \sqrt{2}}{3} \sqrt{\left|\frac{\gamma_{c}\left(\theta, \kappa_{c}(\theta)\right)^{3}}{U^{\prime \prime \prime}\left(\theta, \kappa_{c}(\theta)\right)}\right|},
$$

as a function of $\kappa_{c}(\theta)$, see Fig. 5. The global behavior is given by the 0th order approximation, see prefactor in Eq. (30). The second order approximation, where we expanded all expressions near $\kappa=\pi$, works well near $\kappa=\pi$, but deviates substantially from the real solution given by the 0th order approximation when $(\kappa \bmod 2 \pi) \rightarrow 0$ or $\rightarrow 2 \pi$. In Fig. 5(b), as $\kappa$ increases, one sees two branches, given by Eq. (33). One of them corresponds to the negative critical current branch, $c f$. Fig. 4(b), another to the positive one. At $\kappa$ slightly larger than $\pi$ the positive $\gamma_{c}(\kappa)$ branch crosses zero, see Fig. 4(b), so that we see that the prefactor also vanishes at this point, see Fig. 5(b). Then, at somewhat larger $\kappa$ both mentioned branches join, see Fig. 4(b). At this point the prefactor diverges, see Fig. 5(b). The other two branches in Fig. 5(b) show similar behavior.

\section{Eigenfrequency}

In general, the eigenfrequency of phase oscillations around one of the static solution $\theta_{c} \pm \delta \theta$, see Eq. (26), is given by

$$
\omega_{0}^{2}=U^{\prime \prime}\left(\theta_{c} \pm \delta \theta\right)=U^{\prime \prime}\left(\theta_{c}\right) \pm U^{\prime \prime \prime}\left(\theta_{c}\right) \delta \theta .
$$

The first term vanishes because of Eq. (24). So, using Eq. (26) we get

$$
\omega_{0}^{2}=\sqrt{\left|2 \gamma_{c} U^{\prime \prime \prime}\left(\theta_{c}\right)\right|} \delta \gamma^{1 / 2} .
$$

In the 0th approximation $\theta_{c}=\pi / 2, U^{\prime \prime \prime}\left(\theta_{c}\right)=-\gamma_{c 0}$ and we arrive to the well-known result

$$
\omega_{0}^{2}=\gamma_{c 0} \sqrt{2} \delta \gamma^{1 / 2} \text {. }
$$

In the second order approximation we again sweep $\theta$ and make an implicit plot of the $\omega_{0}^{2}$ prefactor

$$
P_{\omega_{0}}^{2}(\theta) \equiv \omega_{0}^{2} / \delta \gamma^{1 / 2}=\sqrt{\left|2 \gamma_{c}\left(\theta, \kappa_{c}(\theta)\right) U^{\prime \prime \prime}\left(\theta, \kappa_{c}(\theta)\right)\right|},
$$




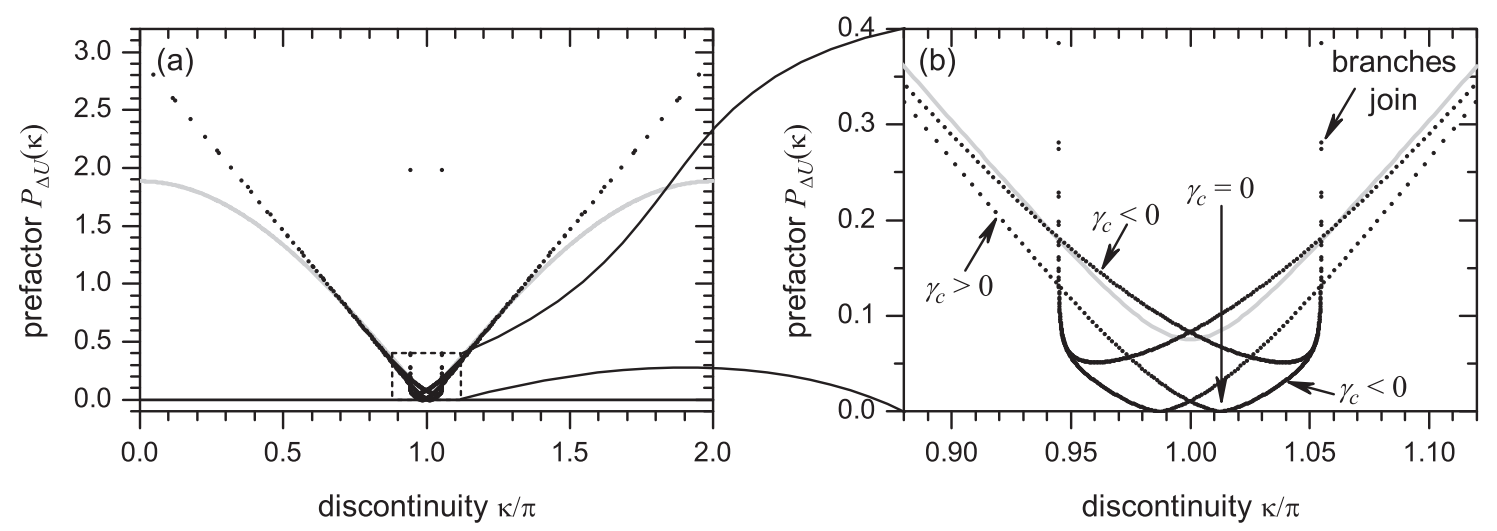

FIG. 5. The energy barrier prefactor $P_{\Delta U}$ given by Eq. (33) vs $\kappa_{c}(\theta)$ (black) and the one calculated using the 0th order approximation Eq. (30) (gray). (a) shows global behavior in the interval $0 \leqslant \kappa \leqslant 2 \pi$, while (b) shows the zoom of the area close to $\kappa=\pi$, where multiple solutions appear. Parameters are: $w=0.5, X_{0}=0.04$.

as a function of $\kappa_{c}(\theta)$ given by Eq. (16). The behavior of the eigenfrequency prefactor is shown in Fig. 6. Similar to the energy barrier prefactor, the eigenfrequency prefactor given by Eq. (37) describes the multiple solutions near $\kappa=\pi$ well. However, the 0th approximation is better outside this vicinity.

\section{Escape histogram width in the MQT regime}

The dependences $\Delta U(\delta \gamma, \kappa)$ and $\omega_{0}(\delta \gamma, \kappa)$ allow us to directly calculate not only the escape rate but also the width $\sigma$ of the escape histogram as a function of $\kappa$. This $\sigma(\kappa)$ dependence can be directly compared with the experimentally measured one. For the sake of simplicity we limit ourselves to the case of MQT, so that the temperature is excluded. The approximate, but rather precise, formula for the histogram width (dispersion) $\sigma$ was derived by Garg [42] in the general case of a particle in a tilted potential. For MQT regime the Garg [42] expression reduces to

$$
\sigma_{\delta \gamma} \propto\left[\frac{P_{\omega_{0}}}{P_{\Delta U}}\right]^{\frac{4}{5}},
$$

where we have omitted ln terms that are much weaker than power terms. We use a $\propto$ sign as we are interested not in the width itself but in its scaling as a function of $\kappa$. This $\sigma$ is a dispersion of $\delta \gamma$ defined above, i.e., it assumes that the critical current is equal to 1 . If the critical current is equal to $\gamma_{c}$, the sigma (measured in the same units as $\gamma_{c}$ ) is

$$
\sigma(\kappa)=\left[\frac{P_{\omega_{0}(\kappa)}}{P_{\Delta U(\kappa)}}\right]^{\frac{4}{5}}\left|\gamma_{c}(\kappa)\right| .
$$

When $X_{0} \gg w^{2} / 3$, or $\kappa \bmod 2 \pi$ is not very close to $\pi$, we can use the 0th order approximation. In this case, by substituting the prefactors from Eq. (30) and Eq. (36) into Eq. (39), we get

$$
\sigma_{0}(\kappa) \propto \gamma_{c 0}^{3 / 5}(\kappa)
$$

This dependence is shown by the gray line in Fig. 7 .

For small $X_{0} \lesssim w^{2} / 3$ and $\kappa \bmod 2 \pi$ in the vicinity of $\pi$, we have to use the second order formulas. Again by substituting prefactors from Eq. (33) and Eq. (37) into Eq. (39) we obtain $\sigma(\theta)$, which we plot vs $\kappa_{c}(\theta)$ as a parametric plot, see Fig. 7. One can see that $\sigma$ vanishes at the bifurcation point where the two branches join. Note also that at the points $\kappa=\kappa_{z}$ where $\gamma_{c}(\kappa)$ vanishes (crosses zero), i.e., $\gamma_{c} \propto \kappa_{z}+\delta \kappa$ is linear, both $P_{\Delta U} \propto \delta \kappa^{3 / 2}$ and $P_{\omega_{0}} \propto \delta \kappa^{1 / 4}$ vanish, however $\sigma \propto$ const does not have zero or any other peculiarity at these points, as can be seen from Eq. (39).

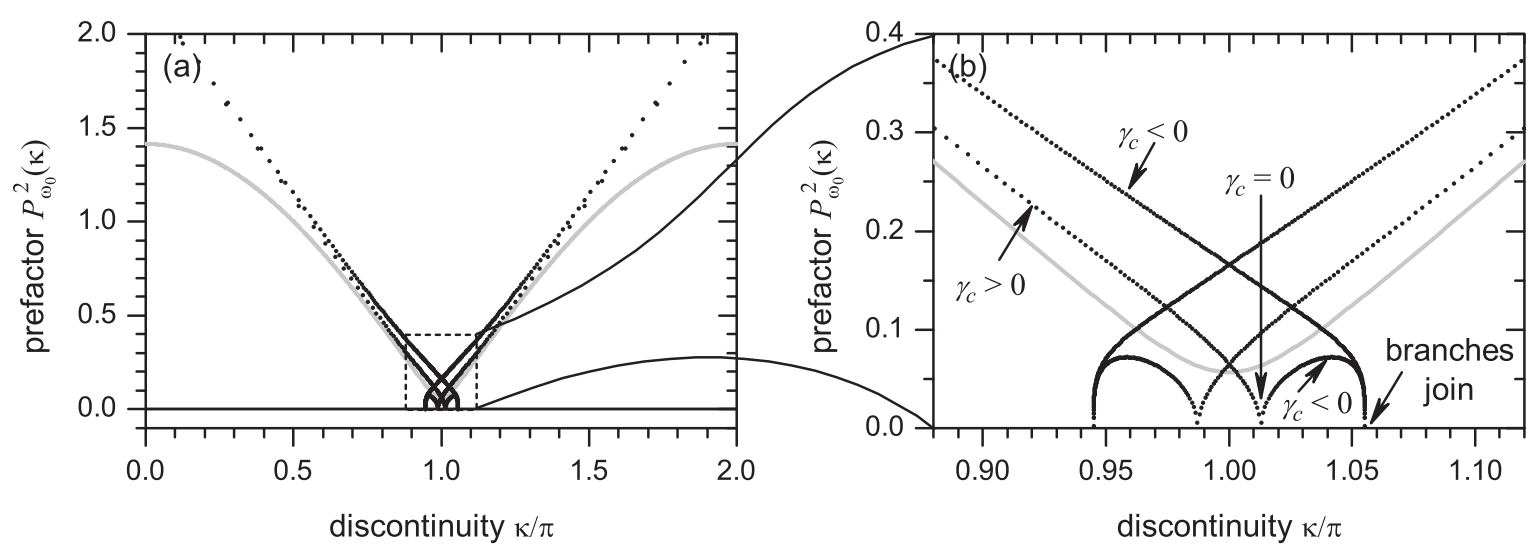

FIG. 6. The eigenfrequency prefactor $P_{\omega_{0}}^{2}$ given by Eq. (37) vs $\kappa_{c}(\theta)$ given by Eq. (16) (black) and the one calculated using the 0th order approximation Eq. (36) (gray). (a) shows global behavior in the interval $0 \leqslant \kappa \leqslant 2 \pi$, while (b) shows the zoom of the area close to $\kappa=\pi$, where multiple solutions appear. Parameters are: $w=0.5, X_{0}=0.04$. 


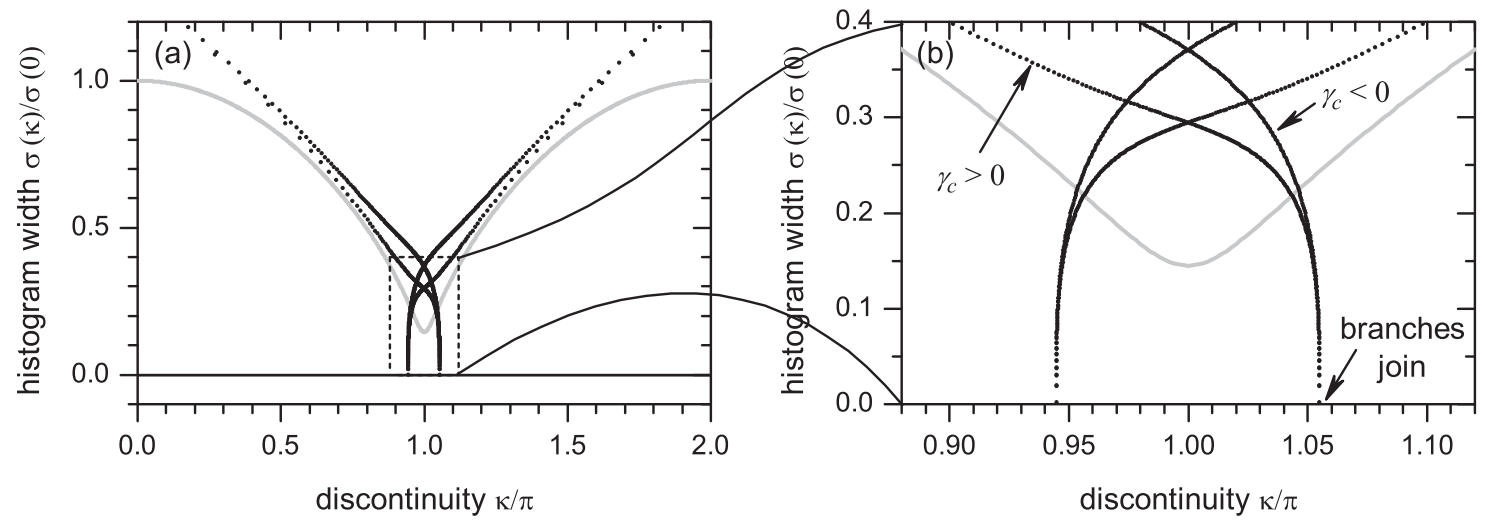

FIG. 7. The width $\sigma(\kappa)$ of the escape current histogram in MQT regime calculated using the 0th order approximation Eq. (40) (gray line) and the second order approximation linearized near $\kappa=\pi$, see Eq. (39). (a) shows the global behavior in the interval $0 \leqslant \kappa \leqslant 2 \pi$, while (b) shows the zoom of the area close to $\kappa=\pi$, where multiple solutions appear. Parameters are: $w=0.5, X_{0}=0.04$.

\section{E. Experimental relevance}

On one hand the range of $\left|X_{0}\right|<w^{2} / 3\left(\left|x_{0}\right|<w^{3} / 3\right)$, required to create a $\varphi \mathrm{JJ}$ near $\kappa=\pi$, is very tiny. This was already pointed out in the previous works $[17,21,23,39,40]$. This makes it very difficult to controllably fabricate the desired $x_{0}$ - a small technological shift can drastically change the junction.

However even a nominally symmetric junction $\left(X_{0}=0\right)$, due to a tiny technological misalignment can get $X_{0} \neq 0$. As a result an experimental $\left|\gamma_{c}(\kappa)\right|$ curve exhibits asymmetric minima for positive and negative bias current, as in Fig. 4. Also, in experiment it should be easy to conclude whether the asymmetry $\left|X_{0}\right|$ is smaller than $w^{2} / 3$ (and we deal with a $\pm \varphi$ $\mathrm{JJ}$ ) or larger (and we deal with single state $\varphi_{0} \mathrm{JJ}$ ). In the former case the $\gamma_{c}(\kappa)$ dependence should have a cusplike minimum with branch crossing, while in the latter case the minimum will be smooth.

\section{CONCLUSIONS}

We have derived an effective model, which describes a short JJ with a phase discontinuity $\kappa$ at an arbitrary point $x_{0}$ along its width. This model reduces the system considered here to a pointlike $\mathrm{JJ}$ with an unconventional CPR. One can relatively easy obtain all desired characteristics of such a pointlike JJ.
For example, we have found a rich variety of the ground states: $\psi=0$ (for $\kappa=0$ ), $\psi=\varphi_{0}$ (for discontinuity well off the middle of the JJ or for $\kappa$ not near $\kappa=\pi$ ), degenerate $\psi=\varphi_{0} \pm \varphi$ (for $\kappa=\pi$ and small $\left|X_{0}\right|<w^{2} / 3$ ), and, finally, not degenerate $\psi=\psi_{1}$ and $\psi=\psi_{2}$ (for $\kappa$ slightly off $\pi$ and small $\left.\left|X_{0}\right|<w^{2} / 3\right)$ ground states. Interestingly, in the latter two cases such junction, if used as a phase battery, will provide two distinct values of the persistent current that are not sign reversal symmetric. We also calculated the dependence of the critical current of such a JJ as a function of $\kappa$ and found multiple branches close to $\kappa=\pi$, corresponding to $\varphi_{0} \pm \varphi$ or $\psi_{1}, \psi_{2}$ states. The value of the critical currents depends on the $\mathrm{JJ}$ state, which can be used to distinguish the states experimentally, similarly to $\pm \varphi$ JJ [23]. Further, we have calculated the behavior of the energy barrier and eigenfrequency close to the critical current, which allow us to make estimations of the width $\sigma$ of the switching current histogram in the regime of macroscopic quantum tunneling and direct comparison with experiment.

\section{ACKNOWLEDGMENT}

This work was supported by EU-FP6-COST Action MP1201 and Deutsche Forschungsgemeinschaft (DFG project Go-1106/5 and project A5 within SFB/TR-21).

\section{APPENDIX A: DERIVATION OF THE AVERAGED CPR}

We use the following phase ansatz

$$
\phi(x)=\phi_{0}+\left\{\begin{array}{ll}
-\frac{\kappa}{2}+A_{L}\left(x-x_{0}\right)+\frac{1}{2} B_{L}\left(x-x_{0}\right)^{2}+\frac{1}{3} C_{L}\left(x-x_{0}\right)^{3}+\frac{1}{4} D_{L}\left(x-x_{0}\right)^{4}, & -w<x<x_{0} \\
+\frac{\kappa}{2}+A_{R}\left(x-x_{0}\right)+\frac{1}{2} B_{R}\left(x-x_{0}\right)^{2}+\frac{1}{3} C_{R}\left(x-x_{0}\right)^{3}+\frac{1}{4} D_{R}\left(x-x_{0}\right)^{4}, & x_{0}<x<+w
\end{array},\right.
$$

which corresponds to the phase discontinuity at $x=x_{0}$ and a Taylor expansion of the phase $\phi(x)$ in the left and right region (subscripts $L$ and $R$ ) relative to the discontinuity $x_{0}$.

In statics, the phase $\phi(x)$ should satisfy the Ferrel-Prange equation $[43,44]$

$$
\gamma=\sin (\phi)-\phi^{\prime \prime}
$$

subject to boundary conditions at the edges $x= \pm w$, corresponding to zero applied magnetic field, and the field continuity at $x=x_{0}$ :

$$
\begin{aligned}
\phi^{\prime}(-w) & =0 ; \\
\phi^{\prime}(+w) & =0 ; \\
\phi^{\prime}\left(x_{0}-0\right) & =\phi^{\prime}\left(x_{0}+0\right) .
\end{aligned}
$$

The prime denotes $\partial / \partial x$. Below, we use the junction halfwidth $w$ as a small parameter and develop a perturbation theory with respect to $w$. 


\section{0th approximation in $w$}

We substitute the ansatz Eq. (A1) into the Ferrel-Prange Eq. (A2). After calculating $\phi^{\prime \prime}$, we would like to expand $\sin (\phi)$ with respect to the small parameter $w$. The key point is to make this expansion correctly. For this, we transform the argument of the sine function to explicitly pull out $w$ from all terms. Namely, we define that $A_{L, R}$ and $B_{L, R}$ from ansatz Eq. (A1) depend on $w$ as

$$
\begin{gathered}
x-x_{0}=\Delta X w ; \\
A_{L, R}=a_{L 1, R 1} w ; \\
B_{L, R}=b_{L 0, R 0} .
\end{gathered}
$$

Here and below the subscripts separated by a comma mean that it is actually two equations: one is obtained by taking the first subscript in the whole equation; the second equation is obtained by taking the second subscript in the whole equation. The higher order $C$ and $D$ terms from Eq. (A1) are not relevant in 0th approximation. Initially, the scaling of $A$ and $B$ with $w$ is actually not obvious, but later we will see that the scaling given by Eqs. (A5) and (A6) is consistent. After the above substitution we expand $\sin (. .$.$) in Eq. (A2) relative to w$, keeping only constant terms (neglecting $O(w)$ and smaller). We arrive at the following expression(s).

$$
b_{L 0, R 0}=\sin \left(\phi_{0} \mp \frac{\kappa}{2}\right)-\gamma_{0},
$$

where $\gamma=\gamma_{0}$ in our 0th approximation. From the Eqs. (A7) it is obvious that $B$ scales $\sim w^{0}$ as written in Eq. (A6). From the boundary conditions Eq. (A3) we have

$$
\begin{aligned}
& a_{L 1}=\left(X_{0}+1\right) b_{L 0} ; \\
& a_{R 1}=\left(X_{0}-1\right) b_{L 0} ; \\
& a_{L 1}=a_{R 1},
\end{aligned}
$$

where $X_{0}=x_{0} / w$. It is Eqs. (A8a) and (A8b) where it becomes obvious that $A_{L, R} \sim w$, as it was correctly written in Eq. (A5), otherwise the 1.h.s. and the r.h.s. would have different orders in $w$. By substituting $b_{L 0, R 0}$ from Eqs. (A7) into Eqs. (A8a) and (A8b) and then $a_{L 1}$ and $a_{R 1}$ from Eqs. (A8a) and (A8b) into Eq. (A8c), we finally get the current-phase relation

$$
\gamma_{0}\left(\phi_{0}\right)=\cos \left(\frac{\kappa}{2}\right) \sin \left(\phi_{0}\right)-X_{0} \sin \left(\frac{\kappa}{2}\right) \cos \left(\phi_{0}\right) .
$$

\section{2nd order approximation}

In the next order $\left(\sim w^{2}\right)$ approximation we use all the terms in ansatz (A1) to substitute into the Eq. (A2). After calculating $\phi^{\prime \prime}$, we explicitly extract $w$ from all terms using the following substitutions

$$
\begin{aligned}
A_{L, R} & =a_{L 1, R 1} w+a_{L 3, R 3} w^{3} ; \\
B_{L, R} & =b_{L 0, R 0}+b_{L 2, R 2} w^{2} ; \\
C_{L, R} & =c_{L 1, R 1} w \\
\gamma & =\gamma_{0}+w^{2} \gamma_{2} .
\end{aligned}
$$

Here $a_{L 1, R 1}, b_{L 0, R 0}$ and $\gamma_{0}$ are from the 0 th order approximation and $a_{L 3, R 3}$ and $b_{L 2, R 2}$ are the next order corrections. Other powers, e.g., $a_{L 2, R 2}=b_{L 1, R 1}=0$. After the above substitution we expand $\sin (. .$.$) in Eq. (A2) relative to w$, keeping only terms $\sim O\left(w^{2}\right)$ and larger [neglecting $O\left(w^{3}\right)$ and smaller]. We arrive at the second order polynomial in $\Delta X$ equal to zero. Obviously, it can be equal to zero for any $\Delta X$ only if each coefficient in front of $\Delta X^{2}, \Delta X$ and constant are all equal to zero. We, thus, obtain

$$
\begin{aligned}
\Delta X^{0}: b_{L 2, R 2} & =-\gamma_{2} \\
\Delta X^{1}: c_{L 1, R 1} & =\frac{1}{2} a_{L 1, R 1} \cos \left(\phi_{0} \mp \frac{\kappa}{2}\right) \\
\Delta X^{2}: D_{L, R} & =\frac{1}{6} b_{L 0, R 0} \cos \left(\phi_{0} \mp \frac{\kappa}{2}\right) .
\end{aligned}
$$

As a next step we substitute the ansatz (A1) into the boundary conditions (A3). Then we substitute the definitions (A10), cancel the terms from 0th order approximation (if any), substitute $c_{L 1, R 1}, D_{L, R}$ and $b_{L 2, R 2}$ from Eqs. (A11) and obtain

$$
\begin{aligned}
& a_{L 3}=-\frac{b_{L 0}}{3} \cos \left(\phi_{0}-\frac{\kappa}{2}\right)\left(X_{0}+1\right)^{3}-\gamma_{2}\left(X_{0}+1\right) \\
& a_{R 3}=-\frac{b_{R 0}}{3} \cos \left(\phi_{0}+\frac{\kappa}{2}\right)\left(X_{0}-1\right)^{3}-\gamma_{2}\left(X_{0}-1\right)
\end{aligned}
$$

$$
a_{L 3}=a_{R 3} .
$$

By substituting Eqs. (A12a) and (A12b) into Eq. (A12c) we finally obtain

$$
\gamma_{2}=\frac{1}{6} \cos \left(\phi_{0}+\frac{\kappa}{2}\right) b_{R 0}-\frac{1}{6} \cos \left(\phi_{0}-\frac{\kappa}{2}\right) b_{L 0} .
$$

Finally, we substitute $b_{L 0, R 0}$ from Eq. (A7) (0th approximation) and obtain the final expression for the second order correction to the current

$$
\begin{aligned}
\gamma_{2}\left(\phi_{0}\right)= & \frac{1}{6}\left(1-X_{0}^{4}\right) \sin ^{2}\left(\frac{\kappa}{2}\right) \sin \left(2 \phi_{0}\right) \\
& +\frac{1}{6} X_{0}\left(1-X_{0}^{2}\right) \sin (\kappa)\left[1+\cos \left(2 \phi_{0}\right)\right],
\end{aligned}
$$

\section{From $\phi_{0}$ to the average phase $\psi$}

Up to now both the 0th order CPR Eq. (A9) and the second order CPR Eq. (A9) are given as functions of the $\phi_{0}$, while our aim is to express those as a function of the average phase $\psi$. To find $\psi$, we substitute ansatz Eq. (A1) into Eq. (1), integrate on each interval, substitute the definitions of $A, B, C, D$ from Eqs. (A10), then substitute expressions for $a, b, c, D$ and $\gamma_{0}$ from Eqs. (A7), (A11), (A12) and (A9). Then we keep only the terms $\sim O\left(w^{2}\right)$ and larger, after some simplifications arrive at

$$
\psi=\phi_{0}-\frac{\kappa}{2} X_{0}+w^{2} \frac{2}{3} X_{0}\left(1-X_{0}^{2}\right) \sin \left(\frac{\kappa}{2}\right) \cos \left(\phi_{0}\right) .
$$


Our aim is to invert this expression, i.e., to express $\phi_{0}(\psi)$ to substitute to CPR and obtain $\gamma(\psi)$. We again act following the perturbation theory with respect to the small parameter $w$. In the 0th approximation

$$
\phi_{0}^{(0)}=\psi+\frac{\kappa}{2} X_{0} \equiv \theta .
$$

Here we introduced the angle $\theta$, which makes expressions more compact.

In the next (second) approximation $\phi_{0}=\phi_{0}^{(0)}+w^{2} \phi_{0}^{(2)}$. By substituting this into Eq. (A15) and expanding up to $O\left(w^{2}\right)$, we obtain $\phi_{0}^{(2)}$ and therefore

$$
\phi_{0}(\psi)=\theta-w^{2} \frac{2}{3} X_{0}\left(1-X_{0}^{2}\right) \sin \left(\frac{\kappa}{2}\right) \cos (\theta) .
$$

Finally, we substitute this into expressions (A9) and (A14), expand up to $O\left(w^{2}\right)$ and after some simplifications obtain

$$
\begin{aligned}
\gamma(\theta)= & \cos \left(\frac{\kappa}{2}\right) \sin (\theta)-X_{0} \sin \left(\frac{\kappa}{2}\right) \cos (\theta) \\
& +Q \sin ^{2}\left(\frac{\kappa}{2}\right) \sin (2 \theta),
\end{aligned}
$$

where, for the sake of brevity, we have introduced

$$
Q \equiv \frac{1}{6} w^{2}\left(1-X_{0}^{2}\right)^{2}
$$

\section{APPENDIX B: COMPARISON WITH THE PREVIOUS RESULTS}

For $\kappa=\pi$ we expect the result given by Eqs. (3), (4), and (5) to be similar to those previously obtained for an asymmetric $0-\pi \mathrm{JJ}$ [39]. To compare both results, first, we have to convert the phases. In Ref. [39] the phase is continuous, while in our case it is has a discontinuity. Instead of the discontinuous phase $\phi(x)$ we can introduce the continuous phase $\mu(x)$, which behaves exactly like $\phi(x)$, but without a $\kappa$ jump, i.e.,

$$
\phi(x)=\left\{\begin{array}{ll}
\mu(x) & \text { for }-w<x<x_{0} \\
\mu(x)+\kappa & \text { for } x_{0}<x<+w
\end{array} .\right.
$$

Then

$$
\psi \equiv\langle\phi\rangle=\langle\mu\rangle+\left(1-X_{0}\right) \frac{\kappa}{2} .
$$

It is $\langle\mu\rangle$ that is used in Ref. [39] (it is denoted as $\psi$ there). Rewriting our effective CPR in terms of $\langle\mu\rangle$ and taking $\kappa=\pi$, we obtain

$$
\gamma=X_{0} \sin \langle\mu\rangle-Q \sin (2\langle\mu\rangle) .
$$

The quantities such as $\left\langle j_{c}\right\rangle, L_{0}$ and $L_{\pi}$ from Ref. [39] can be expressed in terms of quantities used here as

$$
\begin{gathered}
L_{0}=w+x_{0}=w\left(1+X_{0}\right) ; \\
L_{\pi}=w-x_{0}=w\left(1-X_{0}\right) ; \\
\left\langle j_{c}\right\rangle=\frac{L_{0}-L_{\pi}}{L_{0}+L_{\pi}}=X_{0} .
\end{gathered}
$$

By substituting this into expression (18) of Ref. [39] and taking into account the definition of $\Gamma_{0}$, see Eq. (17) of Ref. [39], we arrive at the CPR (B3) derived here. Thus, for $\kappa=\pi$ the result of Ref. [39] is reproduced exactly. Note however that in Ref. [39] the small parameter is the deviation of the phase from its average value, while in our case the small parameter is $w$. Although, they are related (one expects small deviations for small $w$ ), this relation is not straightforward.

In terms of variables used here

$$
\Gamma_{0}=-\frac{1}{3} w^{2} \frac{\left(X_{0}^{2}-1\right)^{2}}{X_{0}} .
$$

If $\Gamma_{0}<-1$ then we have a $\pm \varphi \mathrm{JJ}$. This means that only for $\left|X_{0}\right|<w^{2} / 3$ one obtains a $\varphi \mathrm{JJ}$ at $\kappa=\pi$.
[1] A. A. Golubov, M. Y. Kupriyanov, and E. Il'ichev, Rev. Mod. Phys. 76, 411 (2004).

[2] A. I. Buzdin, Rev. Mod. Phys. 77, 935 (2005).

[3] L. N. Bulaevskii, V. V. Kuzii, and A. A. Sobyanin, Pis'ma Zh. Eksp. Teor. Fiz. 25, 314 (1977) [JETP Lett. 25, 290 (1977)] .

[4] A. I. Buzdin, L. N. Bulaevskii, and S. V. Panyukov, JETP Lett. 35, 178 (1982).

[5] T. Kontos, M. Aprili, J. Lesueur, F. Genêt, B. Stephanidis, and R. Boursier, Phys. Rev. Lett. 89, 137007 (2002).

[6] M. Weides, M. Kemmler, E. Goldobin, D. Koelle, R. Kleiner, H. Kohlstedt, and A. Buzdin, Appl. Phys. Lett. 89, 122511 (2006).

[7] G. Wild, C. Probst, A. Marx, and R. Gross, Eur. Phys. J. B 78, 509 (2010).

[8] V. V. Ryazanov, V. A. Oboznov, A. Y. Rusanov, A. V. Veretennikov, A. A. Golubov, and J. Aarts, Phys. Rev. Lett. 86, 2427 (2001).

[9] H. Sellier, C. Baraduc, F. Lefloch, and R. Calemczuk, Phys. Rev. B 68, 054531 (2003).

[10] V. A. Oboznov, V. V. Bol'ginov, A. K. Feofanov, V. V. Ryazanov, and A. I. Buzdin, Phys. Rev. Lett. 96, 197003 (2006).
[11] J. W. A. Robinson, S. Piano, G. Burnell, C. Bell, and M. G. Blamire, Phys. Rev. B 76, 094522 (2007).

[12] J. A. van Dam, Y. V. Nazarov, E. P. A. M. Bakkers, S. De Franceschi, and L. P. Kouwenhoven, Nature (London) 442, 667 (2006).

[13] I. V. Krive, A. M. Kadigrobov, R. I. Shekhter, and M. Jonson, Phys. Rev. B 71, 214516 (2005).

[14] A. Buzdin, Phys. Rev. Lett. 101, 107005 (2008).

[15] A. A. Reynoso, G. Usaj, C. A. Balseiro, D. Feinberg, and M. Avignon, Phys. Rev. Lett. 101, 107001 (2008).

[16] M. Alidoust and J. Linder, Phys. Rev. B 87, 060503 (2013).

[17] E. Goldobin, D. Koelle, and R. Kleiner, Phys. Rev. B 91, 214511 (2015).

[18] S. V. Mironov, A. S. Mel'nikov, and A. I. Buzdin, Phys. Rev. Lett. 114, 227001 (2015).

[19] R. G. Mints, Phys. Rev. B 57, R3221 (1998).

[20] R. G. Mints, I. Papiashvili, J. R. Kirtley, H. Hilgenkamp, G. Hammerl, and J. Mannhart, Phys. Rev. Lett. 89, 067004 (2002).

[21] A. Buzdin and A. E. Koshelev, Phys. Rev. B 67, 220504(R) (2003). 
[22] E. Goldobin, D. Koelle, R. Kleiner, and A. Buzdin, Phys. Rev. B 76, 224523 (2007).

[23] H. Sickinger, A. Lipman, M. Weides, R. G. Mints, H. Kohlstedt, D. Koelle, R. Kleiner, and E. Goldobin, Phys. Rev. Lett. 109, 107002 (2012).

[24] S. V. Bakurskiy, N. V. Klenov, T. Y. Karminskaya, M. Y. Kupriyanov, and A. A. Golubov, Supercond. Sci. Technol. 26, 015005 (2013).

[25] D. M. Heim, N. G. Pugach, M. Y. Kupriyanov, E. Goldobin, D. Koelle, and R. Kleiner, J. Phys. Condens. Matter 25, 215701 (2013).

[26] E. Goldobin, R. Kleiner, D. Koelle, and R. G. Mints, Phys. Rev. Lett. 111, 057004 (2013).

[27] T. Ortlepp, Ariando, O. Mielke, C. J. M. Verwijs, K. F. K. Foo, H. Rogalla, F. H. Uhlmann, and H. Hilgenkamp, Science 312, 1495 (2006).

[28] M. I. Khabipov, D. V. Balashov, F. Maibaum, A. B. Zorin, V. A. Oboznov, V. V. Bolginov, A. N. Rossolenko, and V. V. Ryazanov, Supercond. Sci. Technol. 23, 045032 (2010).

[29] E. Goldobin, H. Sickinger, M. Weides, N. Ruppelt, H. Kohlstedt, R. Kleiner, and D. Koelle, Appl. Phys. Lett. 102, 242602 (2013).

[30] A. K. Feofanov, V. A. Oboznov, V. V. Bol'ginov, J. Lisenfeld, S. Poletto, V. V. Ryazanov, A. N. Rossolenko, M. Khabipov, D. Balashov, A. B. Zorin, P. N. Dmitriev, V. P. Koshelets, and A. V. Ustinov, Nat. Phys. 6, 593 (2010).
[31] R. F. Voss and R. A. Webb, Phys. Rev. Lett. 47, 265 (1981).

[32] T. Bauch, F. Lombardi, F. Tafuri, A. Barone, G. Rotoli, P. Delsing, and T. Claeson, Phys. Rev. Lett. 94, 087003 (2005).

[33] S.-X. Li, W. Qiu, S. Han, Y. F. Wei, X. B. Zhu, C. Z. Gu, S. P. Zhao, and H. B. Wang, Phys. Rev. Lett. 99, 037002 (2007).

[34] A. V. Ustinov, Appl. Phys. Lett. 80, 3153 (2002).

[35] B. A. Malomed and A. V. Ustinov, Phys. Rev. B 69, 064502 (2004).

[36] E. Goldobin, A. Sterck, T. Gaber, D. Koelle, and R. Kleiner, Phys. Rev. Lett. 92, 057005 (2004).

[37] K. Buckenmaier, T. Gaber, M. Siegel, D. Koelle, R. Kleiner, and E. Goldobin, Phys. Rev. Lett. 98, 117006 (2007).

[38] A. Dewes, T. Gaber, D. Koelle, R. Kleiner, and E. Goldobin, Phys. Rev. Lett. 101, 247001 (2008).

[39] E. Goldobin, D. Koelle, R. Kleiner, and R. G. Mints, Phys. Rev. Lett. 107, 227001 (2011).

[40] A. Lipman, R. G. Mints, R. Kleiner, D. Koelle, and E. Goldobin, Phys. Rev. B 90, 184502 (2014).

[41] T. Gaber, E. Goldobin, A. Sterck, R. Kleiner, D. Koelle, M. Siegel, and M. Neuhaus, Phys. Rev. B 72, 054522 (2005).

[42] A. Garg, Phys. Rev. B 51, 15592 (1995).

[43] A. Barone and G. Paternò, Physics and Application of the Josephson Effect (John Wiley and Sons, New York, 1982).

[44] K. K. Likharev, Dynamics of Josephson Junctions and Circuits (Gorden and Breach, Philadelphia, 1986). 